\title{
Targeted high throughput sequencing in clinical cancer Settings: formaldehyde fixed-paraffin embedded (FFPE) tumor tissues, input amount and tumor heterogeneity
}

Martin Kerick ${ }^{1 \dagger}$, Melanie Isau ${ }^{1,2+}$, Bernd Timmermann ${ }^{1}$, Holger Sültmann $^{3}$, Ralf Herwig ${ }^{1}$, Sylvia Krobitsch', Georg Schaefer ${ }^{4,5}$, Irmgard Verdorfer ${ }^{5,6}$, Georg Bartsch ${ }^{4}$, Helmut Klocker ${ }^{4}$, Hans Lehrach ${ }^{1}$ and Michal R Schweiger ${ }^{1 *}$

\begin{abstract}
Background: Massively parallel sequencing technologies have brought an enormous increase in sequencing throughput. However, these technologies need to be further improved with regard to reproducibility and applicability to clinical samples and settings.
\end{abstract}

Methods: Using identification of genetic variations in prostate cancer as an example we address three crucial challenges in the field of targeted re-sequencing: Small nucleotide variation (SNV) detection in samples of formalinfixed paraffin embedded (FFPE) tissue material, minimal amount of input sample and sampling in view of tissue heterogeneity.

Results: We show that FFPE tissue material can supplement for fresh frozen tissues for the detection of SNVs and that solution-based enrichment experiments can be accomplished with small amounts of DNA with only minimal effects on enrichment uniformity and data variance.

Finally, we address the question whether the heterogeneity of a tumor is reflected by different genetic alterations, e.g. different foci of a tumor display different genomic patterns. We show that the tumor heterogeneity plays an important role for the detection of copy number variations.

Conclusions: The application of high throughput sequencing technologies in cancer genomics opens up a new dimension for the identification of disease mechanisms. In particular the ability to use small amounts of FFPE samples available from surgical tumor resections and histopathological examinations facilitates the collection of precious tissue materials. However, care needs to be taken in regard to the locations of the biopsies, which can have an influence on the prediction of copy number variations. Bearing these technological challenges in mind will significantly improve many large-scale sequencing studies and will - in the long term - result in a more reliable prediction of individual cancer therapies.

\section{Background}

According to the world health organization (WHO) malignant neoplasms are the most common cause of death worldwide in 2010 [1]. We now know that human solid tumors, which account for the majority of all human cancers, result from the accumulation of

\footnotetext{
* Correspondence: mschweig@molgen.mpg.de

† Contributed equally

'Max Planck Institute for Molecular Genetics, Innestr. 63-73, 14195 Berlin, Germany

Full list of author information is available at the end of the article
}

numerous genetic and epigenetic alterations that finally lead to the deregulation of protein-encoding genes [2-10].

Previous efforts to identify protein-encoding cancer genes were limited by insufficient technologies to detect genomic alterations on a global scale. Over the last years more advanced technologies such as next generation sequencing (NGS) technologies have been developed to detect the various patterns of mutations and rearrangements in individual cancer genomes revealing 
the complexity of tumor genetics [11]. These NGS technologies promise to bring about a revolution in cancer genomics such that it becomes feasible to describe the complex genetic networks underlying tumors and thus to identify pathomechanisms of tumor progression and therapy resistance [12-16].

In this regard first whole genome sequences have been published. For example, sequencing of a cytogenetically normal acute myeloid leukemia genome has revealed eight somatic mutations [14]. Within a similar range is the profile of a sequenced breast tumor with 32 nonsynonymous somatic mutations [15]. Recently the complete genomes of lung cancer and melanoma cell lines have been analyzed and indicate correlations between DNA repair mechanisms and mutational spectra $[17,18]$.

However, even though the power of next generation sequencing (NGS) technologies is enormous, remarkably few studies on cancer genomes have been published so far. This is mainly due to the fact that NGS is still relatively cost - and time - intensive and that bioinformatics analyses of tumor tissues are not only challenging, but also need a lot of time - this is likely to be the major bottleneck in the future. One solution to these drawbacks is to increase the sequencing output by focusing on coding DNA regions $[11,19,20]$. Several targeted DNA enrichment technologies to reduce sequence complexity are available [21-27]. These technologies have been mainly developed using large amounts of input DNA generated from blood samples. To identify somatic mutations in solid tumors, DNA has to be extracted from tissues; with often limited access and amounts of extracted DNA. Formalin fixed and paraffin embedded (FFPE) tissue samples, which are archived on a routine basis in pathology departments, could render more and rare conditions accessible. Although FFPE tissue was successfully used for low-coverage whole genome sequencing and copy number detection it is not known if it can be taken for SNV and InDel detection after targeted enrichment strategies [28].

Here, we have specifically addressed cancer-relevant technical questions for targeted sequencing in cancer genomics. We investigated whether FFPE tissue material can be used for targeted re-sequencing applications. We further evaluated the reproducibility and uniformity of the experiments and the effect of modifications such as DNA input amounts. Finally we addressed the question whether the heterogeneity of the tumor as seen by a pathologist is reflected by different mutation patterns or copy number alterations, e. g. if the localization of the biopsy matters. For this, we established quality standards for targeted re-sequencing experiments which can be also used for round-robin tests in clinical diagnostics.

\section{Methods}

\section{Prostate tissue collection and preparation}

Frozen and paraffin-embedded prostate tissue samples were obtained from five patients who had undergone radical prostatectomy at the Department of Urology, Innsbruck Medical University [29,30]. Immediately after surgery, the prostate specimens were cooled and sent to the pathologist, who performed a rapid section and isolated a prostate slice that was embedded in Tissue-Tek OCT Compound (Sakura Finetek, Staufen, Germany), snap-frozen in liquid nitrogen, and stored at $-80^{\circ} \mathrm{C}$ until use. Pathological and clinical data were retrieved from the clinical databases and patients health records. The study was approved by the ethics committee at the Innsbruck Medical University and is in compliance with the Helsinki Declaration (UN3174 and AM3174).

\section{Isolation of DNA samples from prostate tissues}

DNA samples were isolated from radical prostatectomy specimens of five patients. For isolation of DNA, $3 \mu \mathrm{m}$ sections of the frozen specimens were prepared and stained with hematoxylin and eosin for pathological analysis and exact localization of the tumors. For each tumor sample, a paired benign (histopathologically normal) counterpart region distant from the tumor focus was identified. Selection of different foci was based on differences of histological and morphological phenotypes and was performed and controlled on the basis of $\mathrm{HE}$ stainings and P63/AMACR double immunostainings. P63 as a basal epithelial cell marker is absent in tumors, and tumor cells are positive for AMACR. In each case the two markers displayed different histopathological gradings, in two cases Gleason patterns $3+4$ in the low grade focus and $4+5$ in the high grade focus, the third case displayed an additional tertiary pattern 5 in the high grade focus. Subsequently, depending on the tumor area, 5-10 consecutive $10 \mu \mathrm{m}$ sections were cut and carefully macro-dissected for isolation of tumor and benign regions, and the tissue pieces were collected in pre-cooled DNase/RNase free $1.7 \mathrm{ml}$ micro-centrifuge tubes (Costar, Corning, MA, USA). The number of consecutive slides used for macrodissection was adjusted in each case to approximately $5-10 \mathrm{~cm}^{2}$ of overall tissue section, which corresponds to approximately 5-10 mg of tissue and yielded between 2 and $9 \mu \mathrm{g}$ of DNA. For DNA isolation, the EZ1 DNA tissue kit (Qiagen, Hilden, Germany) was used and the isolation was performed according to the protocol recommended by the supplier on a BioRobot EZ1 (Qiagen) equipped with the EZ1 tissue card. To increase DNA yield, the solubilization buffer was supplemented with additional $40 \mu \mathrm{l}$ of Proteinase K solution (Roche, Basel Switzerland) and protease digestion was carried out over night at $56^{\circ} \mathrm{C}$ 
with repeatedly mixing during the first hours of incubation. After sample isolation the DNA amount was determined by UV spectroscopy using a Nanodrop instrument (PEQLAB Biotechnology, Erlangen Germany) and the quality was assessed by calculating the A260/280 ratio, which had to be $\geq 1.8$.

For isolation of DNA from paraffin-embedded tissue specimens the EZ1 DNA tissue kit (Qiagen) procedure was slightly modified. Combined sections of each sample were suspended in $200 \mu \mathrm{l}$ of sample extraction buffer G2. Samples were incubated for $5 \mathrm{~min}$ at $75^{\circ} \mathrm{C}$ with vigorous mixing $(1400 \mathrm{rpm})$ on a thermomixer (Eppendorf). Thereafter the incubation temperature was lowered to $56^{\circ} \mathrm{C}$ and $10 \mu \mathrm{l}$ of protease $\mathrm{K}$ solution (Roche) were added. Incubation at $56^{\circ} \mathrm{C}$ with continuous shaking was continued for an hour. During that hour samples were suspended 2-3 times by pipetting up and down several times to facilitate dissolution of the tissue samples. Afterwards additional $40 \mu \mathrm{l}$ of protease $\mathrm{K}$ solution were added and the incubation at $56^{\circ} \mathrm{C}$ was continued over night. On the next morning additional $20 \mu \mathrm{l}$ of protease solution was added and the incubation with shaking continued for 1 hour. Then the samples were centrifuged in a table centrifuge (Eppendorf) at $10000 \mathrm{~g}$ for $1 \mathrm{~min}$ to pellet all insoluble material and the supernatant was transferred to a fresh $2 \mathrm{ml}$ sample tube.

DNA was isolated with an EZ1 BioRobot (Qiagen) equipped with an EZ1 DNA Paraffin Section Card using the EZ1 DNA Tissue Kit according to the instructions for this instrument. At the end of the purification procedure the DNA was eluted in $50 \mu \mathrm{l}$ of RNAse/DNAse free water and the DNA concentration was measured using a nanodrop photometer (Peqlab, Erlangen Germany).

\section{DNA capturing of selected regions (3.9 $\mathrm{Mb}$ and $52 \mathrm{Mb}$ )}

The library preparation was performed according to Agilent's SureSelect protocol for Illumina single end sequencing with slight modifications. In brief, 0.5-3.0 $\mu \mathrm{g}$ of genomic DNA was sheared for $90 \mathrm{sec}$ on a Covaris ${ }^{\mathrm{TM}}$ instrument set (duty cycle 20\%, intensity 5 and 200 cycles per burst). The fragmented DNA (200-300 bp) was re-quantified with the Agilent 2100 Bioanalyzer 7500 chip. The following end repair reaction was performed to generate blunt-end fragments with 5'-phosphorylated ends. For the adapter ligation the "A" bases were added to the 3'-end of the DNA fragment. The adapters (5'GATCGGAAGAGCTCGTATGCCGTCTT CTGCTTG3') and (5'ACACTCTTTCCCTA-CACGACGCTCTTCCGATCT3') were used in a 10:1 molar ratio to raw genomic DNA.

The ligation products were purified and size selected with a range of 200-350 bp by agarose gel electrophoresis at $120 \mathrm{~V}$ for $1 \mathrm{~h}$. The amplification of the library was performed with the Phusion High-Fidelity PCR master mix with HF buffer (Finnzymes) using Illumina PCR primers 1.1

(5'AATGATACGGCGACCACCGAGATCTACACT CTTTCCCTACACGACGCTTCCGATCT3') and 2.1 (5'CAA-GCAGAAGACGGCATACGAGCTCTTCCG ATCT3') for 14 cycles.

For the hybrid selection the libraries were adjusted to $500 \mathrm{ng}$ in $3.4 \mu \mathrm{l} \mathrm{H}_{2} \mathrm{O}$ and added to the SureSelect Block solutions. This mixture was heated at $95^{\circ} \mathrm{C}$ for $5 \mathrm{~min}$ and held for $5 \mathrm{~min}$ at $65^{\circ} \mathrm{C}$. The library was then mixed with the prewarmed hybridization buffer $\left(5 \mathrm{~min}\right.$ at $65^{\circ} \mathrm{C}$ ) and SureSelect oligo capture library $\operatorname{mix}\left(2 \mathrm{~min}\right.$ at $\left.65^{\circ} \mathrm{C}\right)$. After $24 \mathrm{~h}$ incubation at $65^{\circ} \mathrm{C}$, the hybridization mix was added to $500 \mathrm{ng}(50 \mu \mathrm{l})$ of M-280 streptavidin Dynabeads (Invitrogen), and the incubation was continued for $30 \mathrm{~min}$ at room temperature (RT). The beads were pulled down and washed once at RT for 15 min with $500 \mu \mathrm{l}$ of SureSelect wash buffer 1 , followed by three $10 \mathrm{~min}$ washes at $65^{\circ} \mathrm{C}$ with $500 \mu \mathrm{l}$ of prewarmed SureSelect wash buffer 2. Hybrid-selected DNA was eluted with $50 \mu \mathrm{l}$ of Elution buffer and incubated for $10 \mathrm{~min}$ at RT. After the pull down of the beads, the supernatant was transferred to a tube containing $50 \mu \mathrm{l}$ of Neutralization buffer and the samples were desalted and concentrated on a QIAquick MinElute column and subsequently eluted in $30 \mu \mathrm{l}$ Elution buffer. The post amplification step was performed with the Herculase polymerase and the SureSelect GA PCR-Primer-mix for 14 cycles.

\section{Quality control and NGS Sequencing}

Quantification of the SureSelect captured library: Before sequencing, the samples were re-quantified with two methods. First, the size and concentration was checked on the Agilent 2100 Bioanalyzer and in a second step the enrichment efficiency was estimated by qPCR (Applied Biosystems) using a primerset for an enriched exon (fw: ATCCCGGTTGTTCTTCTGTG and rv: TTCTGGCTCTGCTGTAGGAAG) and a primerset in an intron region as a negative control (fw: AGGTTT GCTGAGGAACCTTGA and rv: ACCGAAACATCCT GGCTACAG). In general the CT-values of target and control fragments differed by 6 to 10, thus confirming a very good enrichment of our target regions.

After diluting the captured libraries to $10 \mathrm{nM}$, Genome Analyzer single read flow cells were prepared on the supplied Illumina cluster station and 36 bp single end reads on the Illumina Genome Analyzer IIx platform were generated following the manufacturer's protocol. Images from the instrument were processed using the manufacturer's software to generate FASTQ sequence files. 


\section{Affymetrix SNParray}

Cryo-embedded tissue material was genotyped on the Affymetrix 6.0 array, according to the manufacturer's protocol. Array positions with a quality score (p-value) $<0.01$ were used as a 'gold standard' for the comparison with the sequencing data. Sequencing data positions within the enriched regions were used if their coverage exceeded 3-fold. This generated 6, 127 and 6, 122 positions for cryo and FFPE tissue, respectively, that were eligible for comparison. To determine false positive and false negative rates, we set the array data as standard and distinguished between reference call and SNP call depending on the array data.

\section{Bioinformatics analyses}

Alignment: Raw reads were mapped to the golden path version hg19 using the bwa 0.5.8 alignment tool with default parameters. Sequences were deposited at the European Genome-phenome Archive [EGA: EGAS00001000136]. Enrichment statistics were calculated for target regions extended by $100 \mathrm{bp}$ on either side. A read had to have at least one base within the target region to be evaluated "on target".

Coverage uniformity: The coefficient of variation was calculated for normalized mean coverages per exon. Normalization was done by a fixed factor per tissue sample to adjust the median coverage over all exons to the same level across all samples. For each two way comparison per exon we plotted the mean coverage of the exon with lower coverage on the $\mathrm{x}$-axis. To examine the GC content dependent coverage for FFPE preparations for all exons the GC content was counted and exons were combined according to their GC content in step sizes of $0.1 \%$. The basewise average exon coverage was averaged within each bin.

Normalized coverage-distribution plots were calculated as follows: The mean coverage per exon was divided by the overall mean coverage of all exons as normalized coverage ( $x$-axis). The fraction of bait-covered exons in the genome achieving coverages equal or lower than the overall mean coverage is indicated on the $y$-axis.

Sorted coverage plots: Exons were sorted by their mean coverage and plotted along the $\mathrm{x}$-axis. Coverage was plotted on the y-axis using a $\log 10$ scale.

Variant detection and comparison: Initial SNV and InDel detection was done using samtools 0.1 .8 for each sample separately. Detected SNVs were required to have a Phred-scaled SNV probability greater or equal 20 and the SNV had to be present in at least $15 \%$ of all reads at a given position. A two step procedure was then applied to call the SNVs for comparison. SNVs detected by our criteria in one preparation were then examined in the second preparation to see if the SNV was found in at least one read. Discordant positions were determined by complimentary comparisons: SNVs called in preparation A had not to be found in preparation B or vice versa. Divergent positions for the snap frozen versus FFPE comparison could be stratified into false positive and false negative, assuming the snap frozen preparation as reference. For somatic SNV detection from two biopsies of the same prostate cancer tumor the Phred-scale cutoff was required to be greater or equal to 20 and the SNV was required to be found in both tumor foci in at least 4 reads but not in the corresponding benign tissue with a minimal coverage of 10 fold.

\section{Determination of copy number variations}

After the DNA fragments were mapped aligned DNA read frequencies were determined for chromosomal intervals (bins) of 55-190 Kb. Interval sizes were chosen individually for each chromosome so that a minimal count of 600 reads per bin was achieved to ensure even data variance across the genome. The $\log 2$ ratio of tumor versus benign counts per bin was calculated and normalized by setting the genome wide median of the ratios to zero. To visualize copy number changes we calculated a running median of 20 bins using the lowess function in R. Differences in copy number between the two foci of one tumor were visualized by calculating the difference of the two running median vectors. Differences greater or equal 0.2 were highlighted.

\section{Results}

\section{FFPE tissue can be used for targeted DNA capturing} experiments and SNV detection

Thousands of patient samples are stored in pathology departments as formalin fixed and paraffin embedded (FFPE) tissues and provide an excellent source for molecular genetic studies. Previously we have shown that whole genome sequencing can be performed with this material $[28,31]$.

Since many large-scale sequencing projects are now directed towards exome sequencing strategies, the question remains whether targeted re-sequencing on FFPE tissue might be possible. We therefore divided prostate tissue samples of radical prostatectomy specimens and stored one part as snap frozen tissue blocks, the other as FFPE material (Figure 1, Additional file 1, Methods). We used material from both preservation technologies for DNA extraction and subsequent hybridization for DNA capturing followed by Illumina sequencing of the "whole Exome" target region (52 Mb) as well as for a 3.9 Mb custom designed target region (Table 1 Additional file 2, Table S1). One caveat of next generation sequencing protocols from FFPE material is the high temperature (above $90^{\circ} \mathrm{C}$ ) needed to melt the paraffin, which results in a significant fraction of single stranded 


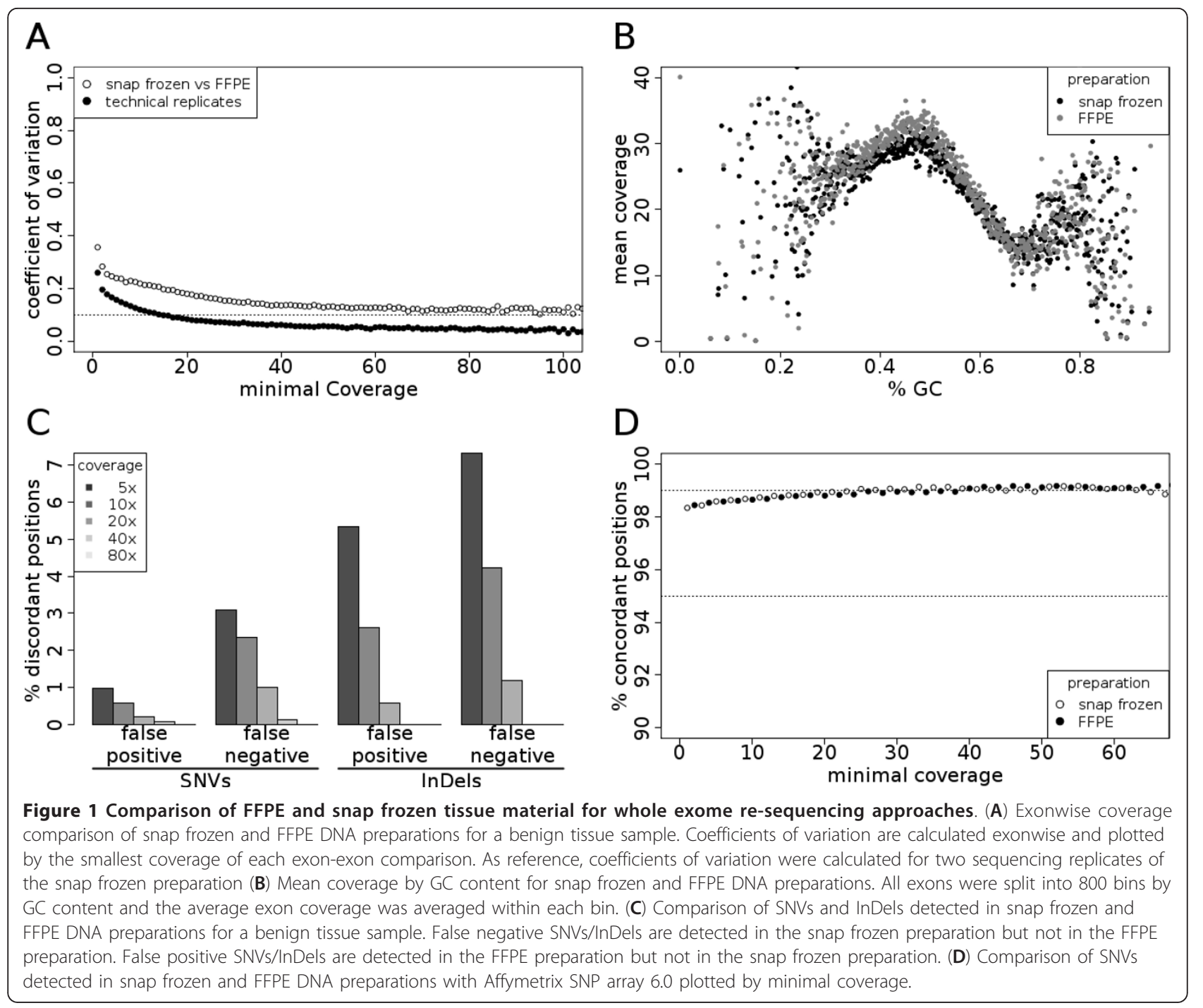

DNA. However, for the subsequent library preparation step double stranded DNA is required. We found that an initial heating step to $75^{\circ} \mathrm{C}$ for $5 \mathrm{~min}$ is sufficient to melt the paraffin and preserve dsDNA. (Detailed protocols for the preparation of DNA, libraries and the capturing are provided in the Material and Method section.) For both DNA materials - snap frozen as well as FFPE stored - we identified an average of $75 \%$ of the sequencing reads located within the whole exome target region and more than $99 \%$ of the regions were captured by at least one read (Table 1, Additional file 2, Table S3). Both preservation technologies have similar coverage profiles and we found a high degree of correlation of enrichment per exon between experiments, which is depicted as coefficient of variation (Figure 1A, Additional file 1 Figure S1A, B). Even though the coefficient of variation is low within the same preparation technology, e.g. snap frozen versus snap frozen and FFPE versus
FFPE, the variation is higher between snap frozen and FFPE. Thus, one should preferably remain within the same tissue preparation technology for one set of experiments (Additional file 1, Figure S1C). It is known that FFPE tissues are prone to spontaneous deamination of guanine and cytosine during the tissue preservation process and/or during storage and that the Illumina sequencing technology is biased for underrepresentation and reduced quality at loci with extreme base compositions [32]. In this regard, we found a slight, but not significant, shift in the GC-dependent coverage profile between the snap-frozen and FFPE tissue (Figure 1B, Additional file 1, Figure S1D).

To assess the effect of coverage depth on the sensitivity and specificity of sequence variant detection, we used genotype calls of an Affymetrix SNP array 6.0 from the cryo material and compared each position to the whole exome sequencing data. For both tissue preparations we 
Table 1 Sequencing statistics

\begin{tabular}{|c|c|c|c|c|c|c|}
\hline ID & sample specification & Target size & \# uniquely aligned reads & $\%$ reads on target & \# of enriched regions & \# of SNVs called $(20 \times)$ \\
\hline Pat1_B & Snap-frozen & $52 \mathrm{Mb}$ & $66,114,467$ & $75.4 \%$ & 200,175 & 18,287 \\
\hline Pat1_B & FFPE & $52 \mathrm{Mb}$ & $71,590,872$ & $74.7 \%$ & 200,032 & 17,810 \\
\hline Pat11_B & Snap-frozen & $3.9 \mathrm{Mb}$ & $28,043,981$ & $62.8 \%$ & 12,274 & 3,000 \\
\hline Pat11_T & Snap-frozen & $3.9 \mathrm{Mb}$ & $18,302,565$ & $71.3 \%$ & 12,226 & 2,511 \\
\hline Pat11_B & FFPE & $3.9 \mathrm{Mb}$ & $9,311,629$ & $67.1 \%$ & 12,175 & 2,577 \\
\hline Pat11_T & FFPE & $3.9 \mathrm{Mb}$ & $15,928,525$ & $73.7 \%$ & 12,254 & 2,883 \\
\hline Pat2_T & $500 \mathrm{ng}$ & $3.9 \mathrm{Mb}$ & $8,760,773$ & $81.1 \%$ & 12,220 & 2,652 \\
\hline Pat2_T & 1500 ng & $3.9 \mathrm{Mb}$ & $9,686,320$ & $79.9 \%$ & 12,243 & 2,848 \\
\hline Pat2_T & 3000 ng & $3.9 \mathrm{Mb}$ & $6,810,410$ & $80.4 \%$ & 12,204 & 2,602 \\
\hline Pat3_B & Benign tissue & $3.9 \mathrm{Mb}$ & $19,617,926$ & $67.7 \%$ & 12,122 & 2,002 \\
\hline Pat3_T & Focus-1 & $3.9 \mathrm{Mb}$ & $28,798,280$ & $68.9 \%$ & 12,227 & 2,328 \\
\hline Pat3_T & Focus-2 & $3.9 \mathrm{Mb}$ & $31,939,154$ & $69.6 \%$ & 11,142 & 2,513 \\
\hline Pat4_B & Benign tissue & $3.9 \mathrm{Mb}$ & $8,878,742$ & $66.2 \%$ & 12,281 & 2,662 \\
\hline Pat4_T & Focus-1 & $3.9 \mathrm{Mb}$ & $8,768,332$ & $64.2 \%$ & 12,288 & 2,645 \\
\hline Pat4_T & Focus-2 & $3.9 \mathrm{Mb}$ & $9,178,790$ & $65.3 \%$ & 12,269 & 2,706 \\
\hline Pat5_B & Benign tissue & $3.9 \mathrm{Mb}$ & $25,957,461$ & $63.2 \%$ & 12,274 & 2,646 \\
\hline Pat5_T & Focus-1 & $3.9 \mathrm{Mb}$ & $65,372,578$ & $64.4 \%$ & 12,261 & 2,388 \\
\hline Pat5_T & Focus-2 & $3.9 \mathrm{Mb}$ & $25,957,461$ & $63.0 \%$ & 11,157 & 2,342 \\
\hline
\end{tabular}

ID: Identification number of the patient's tissue with B for benign tissue and T for tumor tissue.

achieved very similar accuracies above 98\%, even at coverages down to $10 \times$ (Figure 1D).

Next we investigated the reproducibility of single nucleotide variation (SNV) detection in snap frozen versus FFPE tissues. We found 179 (1.2\%) discordant loci investigating positions with at least 20 -fold coverage. The potential artifacts can be grouped into false positives, e.g. a SNV is found in FFPE tissue without evidence in snap frozen tissue and false negative SNVs, where a SNV is found in snap frozen but not in FFPE material. Of the discordant loci we found 149 (0.99\%) potential false positives with all but four that can be explained by processes likely to occur during formalin fixation, as e.g. deamination $(\mathrm{C}>\mathrm{T}, \mathrm{A}>\mathrm{G}, 76$ Loci, $53 \%)$. As false negative SNVs, namely SNVs found in snap frozen preparations but not FFPE preparations, we found 30 loci $(0.2 \%)$ at a coverage level of greater than $20 \times$. We next addressed the question if the differences detected can be overcome using more stringent coverage cutoffs (Figure 1C). While at $40 \times$ coverage $12(0.19 \%)$ discordant loci were found, no discordance is left at $80 \times$ coverage. This also holds true for the custom designed sequencing of a $3.9 \mathrm{Mb}$ region in tumor tissues (Additional file 1, Figure S1E).

In addition to SNVs we also detected insertions and deletions (InDels) and compared InDels detected in snap frozen versus FFPE tissues at a coverage cutoff of $20 \times$. Discordant positions were found more frequent for InDels as opposed to SNVs with $8(1.17 \%)$ loci as false positive and $4(0.58 \%)$ loci as false negative positions. Again, higher coverage levels led to a lower percentage of discordant InDels, with no differences found at a coverage level of $40 \times$ (Figure 1C, Additional file 1, Figure 1F).

\section{Targeted sequence enrichment for small amounts of input DNA}

An important objective of technology development is to lower the amount of input DNA required. To this end, we used the targeted enrichment of $3.9 \mathrm{Mb}$ distributed over 12, 366 independent regions and performed enrichment experiments with three different amounts of DNA (500 ng, $1500 \mathrm{ng}, 3000 \mathrm{ng}$ ) obtained from frozen prostate cancer tissues. We found the enrichment efficiency to be similar for all three DNA amounts: Approximately $80 \%$ of sequencing reads mapped to the target regions and more than $98 \%$ of targets were hit at least once (Table 1, Additional file 2, Table S3). All three preparations had very similar global coverage profiles. In addition we found a high degree of correlation of enrichment per exon between experiments with a coefficient of variation lower than 0.2 at $20 \times$ coverage for all comparisons made (Figure 2A, Additional file 1, Figure S2). The enrichment is highly uniform and reproducible across several experiments (Additional file 1, Figure S2, S3).

Since smaller amounts of DNA might lead to a decreased sample complexity, and thereby to increased data variance, we calculated and visualized the variant/ reference ratio distributions for different DNA 


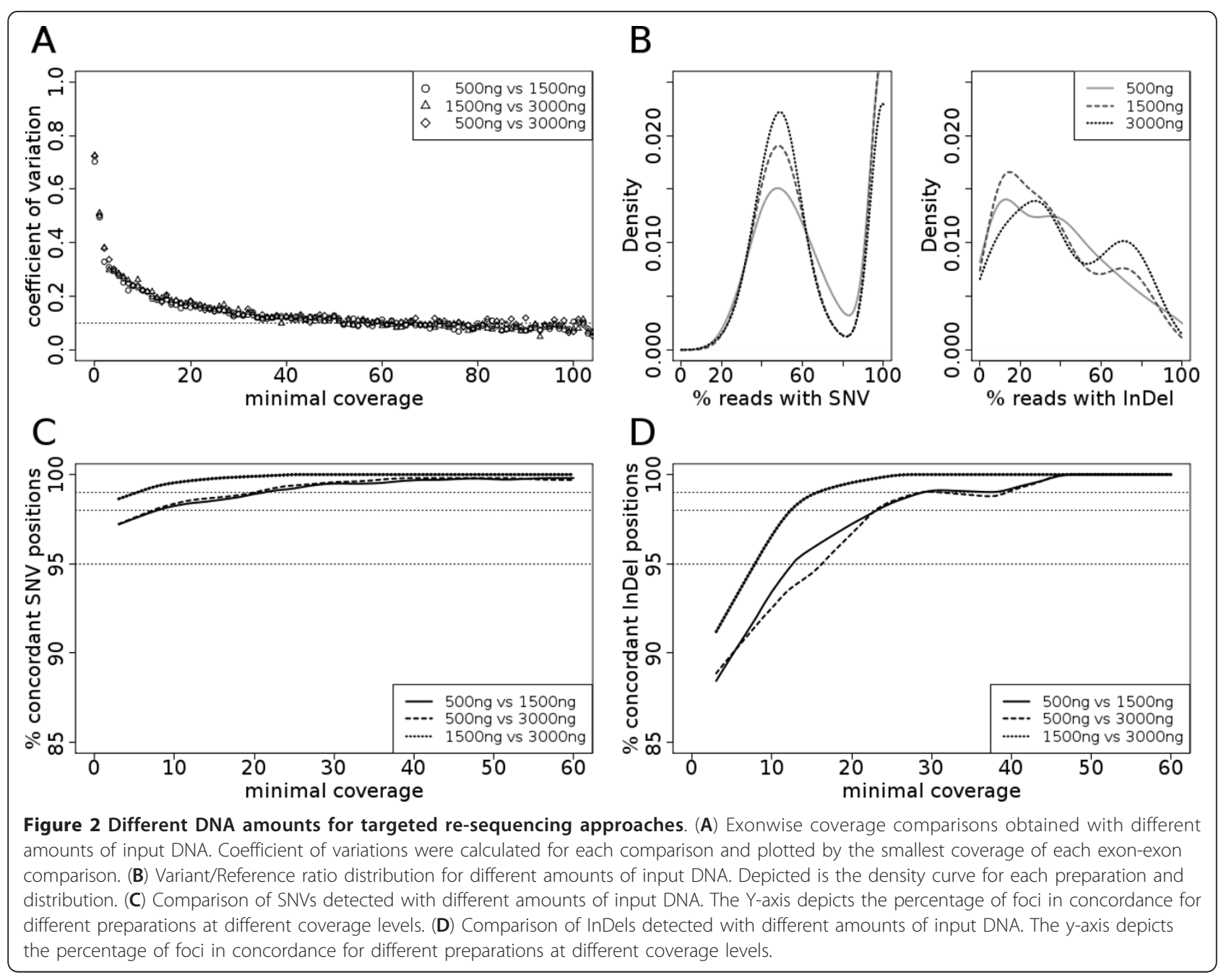

amounts at a coverage level of $50 \times$ for SNVs and InDels (Figure 2B). In an ideal situation a heterozygous position would have $50 \%$ reads showing the variant - a variant/reference ratio of 0.5 . Based on the ratios we find a slightly broader distribution for small amounts of input DNA which is also shifted towards higher ratios. The slightly lowered complexity of the samples with decreased DNA input amounts is also reflected in the number of unique start sites: For $500 \mathrm{ng}$ input material we received $40 \%$ of the expected unique start sites (calculated in relation to the $3.9 \mathrm{Mb}$ target

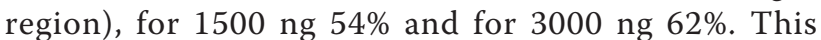
needs to be considered when the input amounts are reduced and when homozygous versus heterozygous gene loci are compared. In comparison to SNV callings, InDels do not follow the expected bimodal distribution for variant/reference ratios but resemble rather a Bernoulli distribution (Figure 2B). Based on these findings, we chose to discard InDels with variant/reference ratios lower than $15 \%$ from further analysis.
For the SNVs and InDel detection we next asked how reproducible they are and how high the coverage needs to be to minimize the error rates. Since all three preparations originated from the same tumor DNA and only the amount of input DNA differed, identical SNVs and InDels should be called. We therefore investigated if SNVs and InDels called for each amount of DNA were found in the other preparations with different amounts of DNA. With a minimum coverage of $3 \times$, we found more than $98 \%$ concordance between two samples for SNVs (Figure 2C). Interestingly, when we looked at SNVs, which had been already annotated in the dbSNP database (referred to as 'known SNVs'), the concordance rates are even higher reaching about $99 \%$ at $3 \times$ coverage. In contrast, when we looked at SNVs which had not been annotated so far ('unknown SNVs'), concordance rates below 55 fold coverages were up to $30 \%$ lower than for 'known' loci (Additional file 1, Figure S4). At coverage rates of $55 \times$ or more, SNV concordances were higher than 98\% for 'known' and 'unknown' 
loci alike. For InDels we found concordance rates of $98 \%$ at above $20 \times$ coverage (Figure 2D), and we observed much smaller differences between known and unknown positions (Additional file 1, Figure S5).

\section{Distinct biopsies from a single tumor have identical somatic SNV profiles in selected prostate cancer} candidate genes, but differ in their copy number patterns A long-standing question of cancer research is whether biopsies are true representatives for the tissue of origin. This is of particular interest since many solid tumors grow as distinct tumor foci. We therefore addressed the questions whether biopsies from prostate tumors are uniform or if they are associated with different mutational patterns or different copy number variations. Prostate cancer is a prototype tumor to address this problem. The majority of these tumors are multifocal and in many cases two or more distinct, locally separated tumor foci can be identified [30,33].

We designed a target gene set of 1121 genes carefully selected by association to prostate cancer, cancer in general and signal transduction pathways. DNA was isolated from two different loci for each tumor in addition to matched benign tissue from frozen radical prostatectomy specimens of three prostate cancer patients. Selection of different foci was based on differences of histological and morphological phenotypes and was performed and controlled on the basis of HE stainings and P63/AMACR double immunostainings. The basal cell marker P63 decorates only benign glands whereas AMACR is a marker for tumor cells. In each case the two tumor foci analyzed displayed different histopathological gradings, in two cases Gleason patterns $3+4$ in the low grade focus and $4+5$ in the high grade focus, the third case displayed an additional tertiary pattern 5 in the high grade focus (See also Table 2 and Additional file 1, Methods).

Targeted enrichment with subsequent sequencing was performed with these nine tissue samples. We found the enrichment efficiency to be very similar for all samples: Approximately $69 \%$ of sequencing reads mapped to the target region and about $99 \%$ of targets were hit at least once (Table 1 and Additional file 2, Table S3). In addition, the coverage profiles were very similar for all patients as demonstrated by the cumulative normalized coverage plot and the coefficients of variation (Figure $3 \mathrm{~A}, \mathrm{~B})$.

For a comparison of the SNV profiles we used a two step procedure for loci covered in both preparations at a minimal coverage level of $20 \times$. First, called SNVs for focus A were required to have at least $15 \%$ of reads containing the SNV. In the second step focus B was then analyzed and a SNV was considered concordant if the SNV was found in at least one read of focus $B$.
Table 2 TMPRSS-ERG Fusion status and somatic mutations of the different foci analysed

\begin{tabular}{|c|c|c|c|}
\hline patient-ID & $\begin{array}{l}\text { Gleason } \\
\text { Score }\end{array}$ & $\begin{array}{l}\text { TMPRSS2-ERG } \\
\text { Fusion }\end{array}$ & $\begin{array}{c}\text { somatic } \\
\text { substitutions }\end{array}$ \\
\hline \multicolumn{4}{|l|}{$\begin{array}{l}\text { Pat3_ } \\
\text { normal }\end{array}$} \\
\hline $\begin{array}{c}\text { Pat3_Focus } \\
1\end{array}$ & $4+5$ & Deletion & $\begin{array}{c}\text { SH3BGR } \\
\text { chr21:40883678 }\end{array}$ \\
\hline $\begin{array}{c}\text { Pat3_Focus } \\
2 \\
\end{array}$ & $3+4$ & no Fusion & \\
\hline \multicolumn{4}{|l|}{$\begin{array}{l}\text { Pat4_ } \\
\text { normal }\end{array}$} \\
\hline $\begin{array}{c}\text { Pat4_Focus } \\
1\end{array}$ & $3+4$ & no Fusion & $\begin{array}{c}\text { SH3BGR } \\
\text { chr21:40883671 }\end{array}$ \\
\hline $\begin{array}{c}\text { Pat4_Focus } \\
2 \\
\end{array}$ & $3+4(5)$ & no Fusion & \\
\hline \multicolumn{4}{|l|}{$\begin{array}{l}\text { Pat5 } \\
\text { normal }\end{array}$} \\
\hline $\begin{array}{c}\text { Pat5_Focus } \\
1\end{array}$ & $3+4$ & no Fusion & $\begin{array}{c}\text { NUB1 } \\
\text { chr7:151053043 }\end{array}$ \\
\hline $\begin{array}{c}\text { Pat5_Focus } \\
2\end{array}$ & $4+5$ & $\begin{array}{l}\text { Deletion and } \\
\text { Insertion }\end{array}$ & \\
\hline
\end{tabular}

Although the SNVs differed substantially between patients, we found no discordant position in any two foci of the same tumor in the three patients at this level of stringency. We also determined the concordance of SNV profiles at smaller coverage levels (Figure 3C). At a minimal coverage of $5 \times$ we observed $0.4 \%$ discordant loci at maximum but this difference is most likely caused by an amplification bias rather than by real differences, since the number of discordant foci quickly diminishes with rising coverage demands. We analyzed small InDels in a similar way and found again higher rates of discordance as compared to SNVs (Figure 3D). Except for one discordant locus found in Patient 5, no discordances were found when higher coverage cutoffs were used. We also investigated potential somatic SNVs by comparing each individual focus with its matched benign tissue. We found one somatic SNV for each of the three patients. This mutation was identified in both tumor foci but not in the benign tissue (Table 2).

In addition to the SNV profiles for the different tumor foci we also investigated the copy number variations within each focus. For this, we generated low-coverage whole genome sequencing profiles for the two tumor foci and the corresponding benign tissue from each patient. We determined potential somatic copy number variations by comparing each tumor focus with the matched benign tissue (Additional file 1, Figure S6). Received copy number variations were then compared between the two foci and the difference was plotted genome-wide (Figure 4). For patient 3 we found clear differences on chromosome 4, 10 and 13 between both foci with regard to CNVs (Additional file 1, Figure S6 


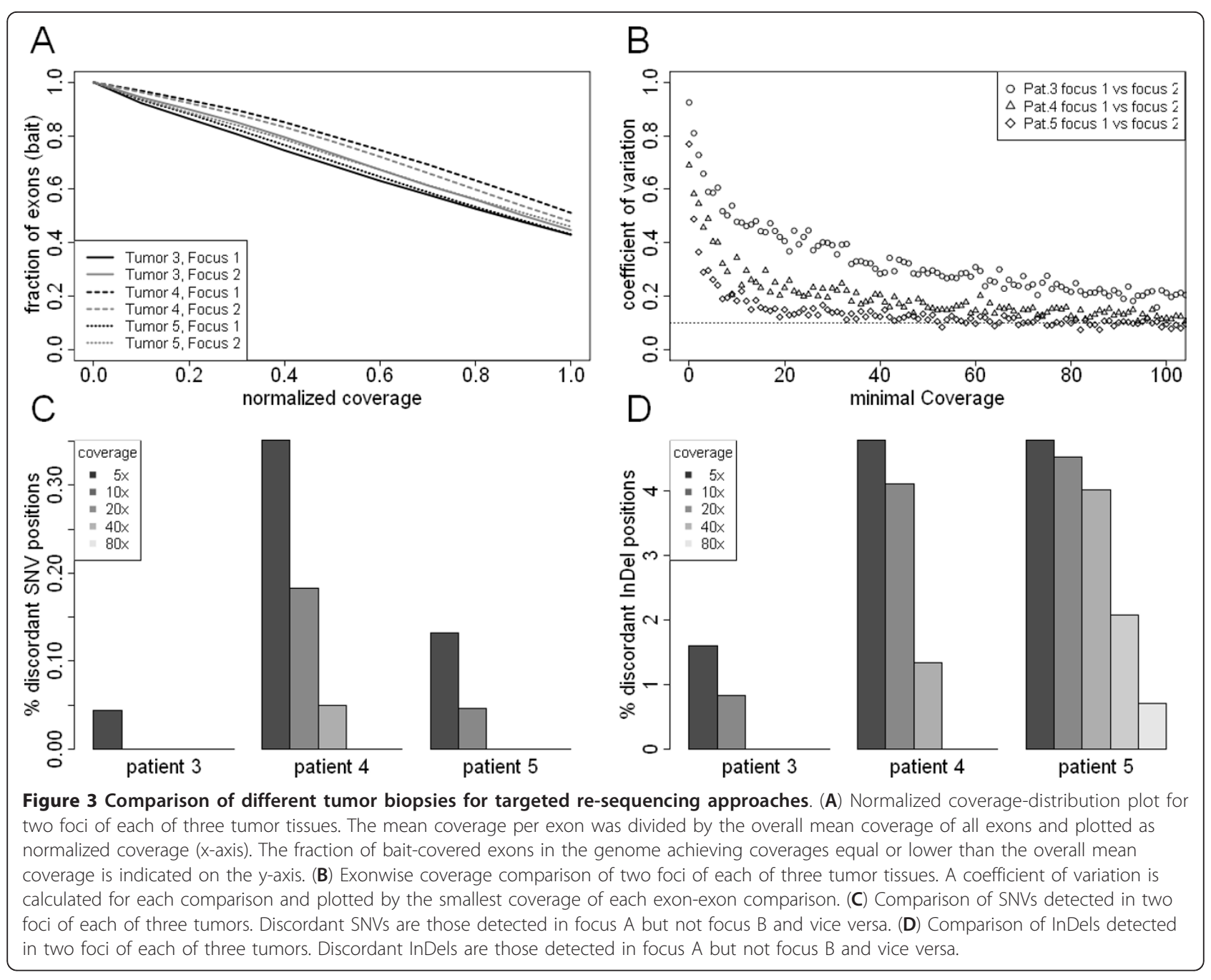

A-C). In comparison, the biopsies taken from patient 4 and 5 seemed more homogeneous as no differences of the CNV profiles were apparent between the two tumor foci. Marked differences for patient 5 are located towards chromosome ends and visual inspection proposed the individual CNVs to be artefacts (Additional file 1, Figure S6 D).

\section{Discussion}

Next-generation technologies such as targeted resequencing platforms are powerful tools for identifying genetic variations in cancer samples. Using prostate cancer as an example, we have assessed the use of different kinds and amounts of tissue samples for identifying genetic variations. In particular, we have investigated three aspects which are frequently addressed from oncologists and pathologists:

The first is whether or not it is possible to use FFPE material in addition to snap frozen material. The use of FFPE material would open up a large collection of tissue samples for molecular studies since most of the materials stored at pathology departments around the world are archived in this way. However, the preparation procedure of FFPE tissue with formaldehyde fixation and long-term storage at room temperature may generate DNA mutations and result in the identification of false SNVs or InDels. We previously showed that it is possible to use FFPE material for copy number analysis of whole genome data, although a higher sequencing capacity is required to achieve a comparable coverage [28]. Now we have extended our studies to targeted enrichment methods and found an uniform enrichment irrespective of the kind of tissue material used. Looking at the numbers of SNVs detected we found $0.98 \%$ false positive SNVs in FFPE preparations at a coverage level of $20 \times$ which can be strongly reduced at higher coverages $(>80 \times)$. Potential false positive SNVs can be explained by processes likely to occur during formalin fixation, like deamination and depurination processes. Our data suggests that the 


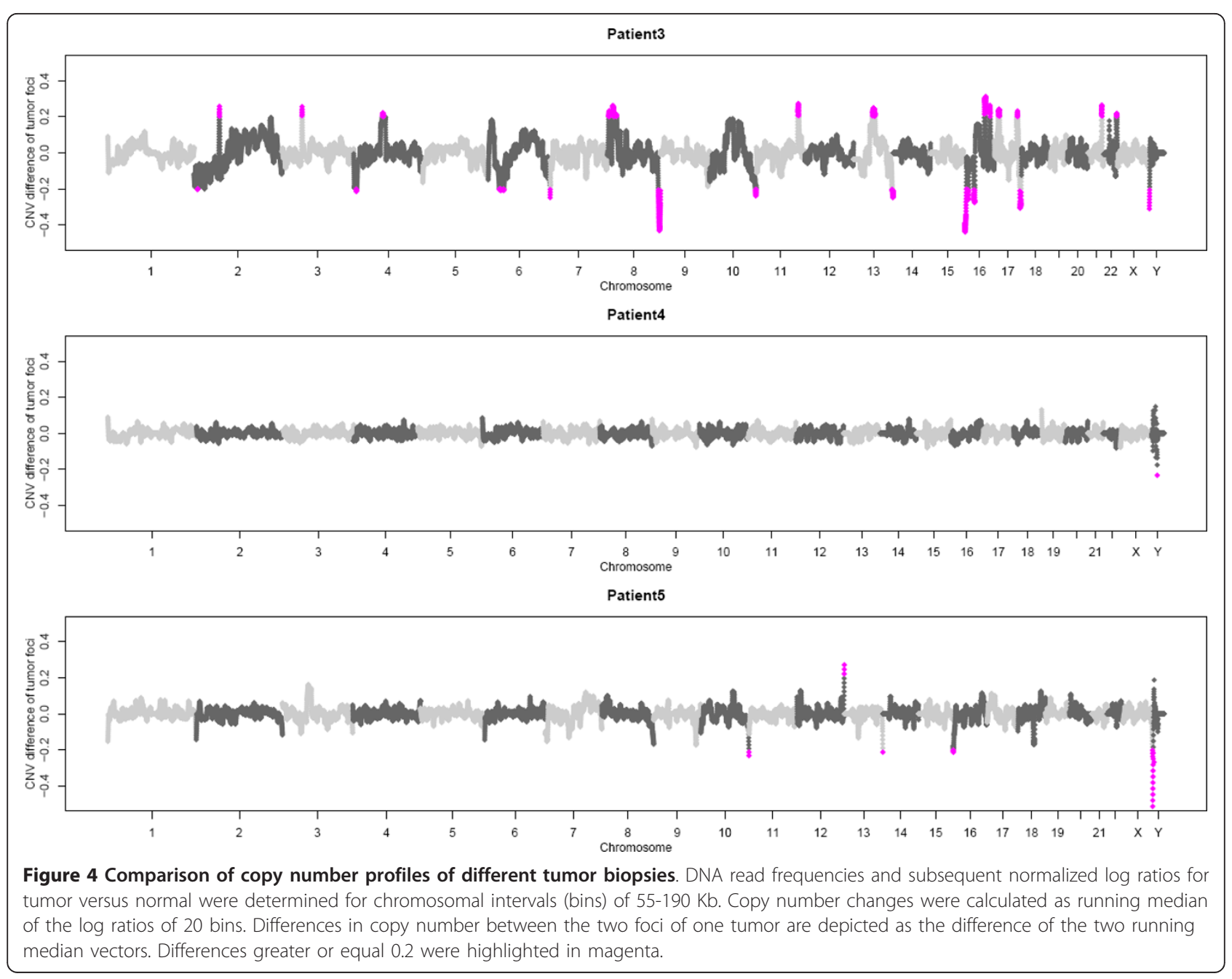

damage done by the FFPE preparation has a random distribution across all DNA fragments and can be corrected by sequencing depth. Since coverage levels of $80 \times$ and higher can easily be reached by targeted resequencing approaches, we recommend to use such high coverages when analyzing FFPE material. The same holds true for false negative SNVs. Keeping in mind that SNV detection is the main focus of DNA sequence analysis in cancer, the detection of small insertions and deletions becomes increasingly important. We therefore investigated if preparation of DNA from FFPE tissue may have an adverse effect on InDel detection. While the relative amount of discordant InDel positions is about 7 times higher than the amount of discordant SNV positions, we observed the same low discrepancy rates at higher coverage levels. Again, no discordance was found at a coverage level of $80 \times$. Taken together, snap frozen tissues remain the preferred source of DNA, but FFPE tissue can be used for SNV and InDel detection instead if the coverage is increased. Furthermore, for certain clinically relevant questions, like for the detection of germline variants, e.g. when for a snap frozen tumor tissue no adequate matching benign tissue material is available, FFPE tissues can be used. In this case, the positive error rate obtained with FFPE material plays an inferior role.

The second methodological issue relates to the amount of material required. Decreasing the input amount of DNA to $500 \mathrm{ng}$ still yielded good enrichment results, an even coverage and a highly reproducible calling of known genetic variants. However, we find increased redundant reads (reads with identical first positions) and a slightly higher variance of variant/reference ratios with decreased amounts of starting material. This suggests that - with these enrichment technologies - the minimal amount of input DNA cannot easily be reduced beyond $500 \mathrm{ng}$. Notably, the comparison among average and high amounts of DNA $(1.5 \mu \mathrm{g}$ vs $3 \mu \mathrm{g})$ performed better than a comparison including the lowest amount of DNA (500 ng). 
While InDels detected show a variant/reference ratio distribution clearly deviating from the expected bimodal distribution and visible differences for the three DNA amounts, InDels are still highly reproducible above a coverage level of $45 \times$ for all amounts of DNA. We conclude that a decrease to $500 \mathrm{ng}$ of input DNA is possible, but the benefit has to be weighed against the high coverage demands and potential challenges to SNV and InDel categorization.

The third challenge presented in our study consists of the heterogeneity of tumor tissue. In order to obtain results representative for the whole tumor, the amount and location of biopsies necessary is unknown. So far, it is not decided whether primary prostate cancers have a multifocal origin and thus are composed of multiple genetically distinct cancer cell clones or not. Currently, an independent clonal nature of multiple foci is considered since healthy men below 40 years frequently show presence of focal histological aberrations [34-36] many of which give rise only to latent prostate cancer, while clonal evolution of a few foci paves the way to clinically detectable disease [33,37-40]. On the other hand, prostate cancer metastases from different locations but from the same patient show a surprisingly similar pattern with regard to copy number alterations [41-43]. Experiments available to address this question include the determination of the DNA ploidy, micro-satellite analysis, c-myc amplifications with FISH, DNA methylation or the TMPRSS2-ERG fusion status on separate tumors within the same prostate [44]. In our hands, using samples derived from different foci within one prostate tumor and performing DNA re-sequencings of prostate cancer relevant genes, we found almost identical distributions of mutations within different foci of the same patient. Notably, SNV profile concordance was $100 \%$ for all three patients at coverage levels above $20 \times$. Even tumor parts with different TMPRSS2-ERG gene fusion status are remarkably identical with regard to small nuclear variations. In addition, focusing on somatic mutations, we find no differences between different tumor foci. However, although we focused on prostate cancer candidate genes, the low number of somatic mutations in prostate cancer and the fact that we only analyzed $\sim 10 \%$ of the exome prohibit a generalized conclusion. Recent studies, such as Taylor et al with 0.31, Kan et al with 0.33, and Berger et al with 0.9 nonsynonymous mutations per $\mathrm{Mb}$, suggest low somatic mutation rates per $\mathrm{Mb}$ for prostate cancer $[8,9,45]$. In line with this somatic mutation frequency we found only one somatic mutation for each of the three patients. The sensitivity of current re-sequencing approaches might further explain the missing focal diversity. Irrespective of the low frequency of somatic mutations we detected in the tumor samples we found large aberrations in copy number. We have used a whole genome re-sequencing approach to detect somatic copy number variations for each focus and compared the two foci from the same tumor. Interestingly, for one patient with clear differences in the TMPRSS2-ERG fusion pattern, we also find significant differences between the two foci, whereas for two other patients no significant CNVs can be detected. Along this line Navin et al. used a modified comparative genomic hybridization $(\mathrm{CGH})$ technology to study the clonal composition of breast tumors and found a large proportion of monogenomic tumors and only a small fraction of tumors with a heterogenomic foci structure [46]. Our results would implicate that the location of biopsies taken within tumors is of minor relevance for the detection of mutations, but plays a major role for the detection of copy number variations. Within this direction, recent publications also suggest that genomic rearrangements are a major genetic factor underlying prostate cancer [47]. Since we did not perform 3D reconstructions of the whole tumors our approach cannot be used to answer the question of multifocal origin of heterogeneous prostate tumors. Even for the estimation of tumor heterogeneity our studies are most likely an underestimation, because we are investigating tissue samples with a complex composition of single cells. Thus, the genetic profiles are the sums over all cells contained within the section and might mask the true tumor heterogeneity. At the moment we are extending our analysis onto a single cell level to further gain insight into the evolutionary architecture of prostate tumors. With this we might be able to pin down the true tumor composition and we might even identify tumor stem cells on a genetic level. However, since we find differences between different biopsies from the same tumor on a copy number level, we can conclude that several biopsies need to be investigated to gain insight into the genomic context of prostate cancers based the overall tumor heterogeneity.

Furthermore, with the technologies described we are now in the progress to extend our analyses to large sample cohorts from pathology departments where we can select tissue specimens from specific clinical studies. This enables us to address clinical relevant questions such as progression and therapy resistance of tumors which is an important step towards the application of targeted re-sequencing approaches as routine diagnostic tools in oncology.

\section{Conclusion}

Illumina sequencing is a powerful tool for large-scale resequencing projects. For clinical applications, in particular for the benefit of cancer patients, several key issues need to be addressed: Tissue material, input amounts 
and reproducibility of the data in regard to tumor heterogeneity. Our optimized protocols guide through each of these issues and provide data for an optimal strategy for the usage in clinical settings. We show that FFPE material can be used with higher coverages as substitution of cryo-frozen tissue and that it is in particular useful for the determination of germline variations when tumor tissues have already been sequenced. Lowering the amount of input material results in an increase of redundant reads and a slightly higher variance of variant/reference ratios, but can be overcome to a certain degree with adequate analysis tools. Finally, the tumor heterogeneity plays an important role for the detection of copy number variations, but is of minor importance for the detection of somatic variations. This implies that the sampling of tumor tissues is of major importance and needs to be taken into consideration for clinical diagnostic purposes.

\section{Additional material}

Additional file 1: Supplementary Methods, Figure Legends S1-S6, Table Legends S1-S3. Figures S1-S6

Additional file 2: Tables S1-S3.

\section{Acknowledgements and Funding}

We would like to thank Nada Kumer, Anna Kosiura, Sonia Paturej, Isabelle Kuehndahl, Ilona Hauenschild, Irma Sottsas and Christof Seifarth for excellent technical assistance.

This work was supported by a grant of the Austrian Nationalstiftung and the Austria Wirtschaftsservice $\mathrm{GmbH}$ in the framework of the IMGuS research program (Institute for Medical Genome Research and Systems Biology, Vienna); and the German Federal Ministry of Education and Research (01GS08105, 01GS08111 and Predict)

\section{Author details \\ ${ }^{1}$ Max Planck Institute for Molecular Genetics, Ihnestr. 63-73, 14195 Berlin, Germany. ${ }^{2}$ Free University Berlin, Department of Biology, Chemistry and Pharmacy, Takustrasse 3, 14195 Berlin, Germany. ${ }^{3}$ Unit Cancer Genome Research, DKFZ German Cancer Research Center and National Center for Tumor Diseases, Im Neuenheimer Feld 460, 69120 Heidelberg, Germany. ${ }^{4}$ Innsbruck Medical University, Department of Urology, Anichstr. 35, A 6020 Innsbruck, Austria. ${ }^{5}$ Innsbruck Medical University, Department of Pathology, Muellerstr. 40, A-6020 Innsbruck, Austria. ${ }^{6}$ Innsbruck Medical University, Department for Medical Genetics, Molecular and Clinical Pharmacology, Division of Human Genetics, Schöpfstraße 4, 6020 Innsbruck, Austria.}

\section{Authors' contributions}

Clinical samples and discussions were provided by GS, IV, GB and HK. MK, $\mathrm{MI}, \mathrm{BT}$ and MRS carried out the experiments, data analyses were done by MK, MI, HK and MRS; HS and MRS conceived and coordinated the project; MK, MI, HS, RH, SK, HK, HL and MRS wrote the paper. All authors read and approved the final version.

\section{Competing interests}

The authors declare that they have no competing interests.

Received: 20 March 2011 Accepted: 29 September 2011 Published: 29 September 2011
References

1. Jemal A, Bray F, Center MM, Ferlay J, Ward E, Forman D: Global cancer statistics. CA Cancer J Clin 61:69-90.

2. Bell DW: Our changing view of the genomic landscape of cancer. J Pathol 2010, 220:231-243.

3. Greenman C, Stephens P, Smith R, Dalgliesh GL, Hunter C, Bignell G, Davies $H$, Teague J, Butler A, Stevens $C$, et al: Patterns of somatic mutation in human cancer genomes. Nature 2007, 446:153-158.

4. Jones S, Zhang X, Parsons DW, Lin JC, Leary RJ, Angenendt P, Mankoo P, Carter $\mathrm{H}$, Kamiyama $\mathrm{H}$, Jimeno A, et al: Core signaling pathways in human pancreatic cancers revealed by global genomic analyses. Science 2008, 321:1801-1806.

5. Parsons DW, Jones S, Zhang X, Lin JC, Leary RJ, Angenendt P, Mankoo P, Carter H, Siu IM, Gallia GL, et al: An integrated genomic analysis of human glioblastoma multiforme. Science 2008, 321:1807-1812.

6. Sjoblom T, Jones S, Wood LD, Parsons DW, Lin J, Barber TD, Mandelker D, Leary RJ, Ptak J, Silliman N, et al: The consensus coding sequences of human breast and colorectal cancers. Science 2006, 314:268-274.

7. Wood LD, Parsons DW, Jones S, Lin J, Sjoblom T, Leary RJ, Shen D, Boca SM, Barber T, Ptak J, et al: The genomic landscapes of human breast and colorectal cancers. Science 2007, 318:1108-1113.

8. Taylor BS, Schultz N, Hieronymus H, Gopalan A, Xiao Y, Carver BS, Arora VK, Kaushik $P$, Cerami E, Reva B, et al: Integrative genomic profiling of human prostate cancer. Cancer Cell 2010, 18:11-22.

9. Kan Z, Jaiswal BS, Stinson J, Janakiraman V, Bhatt D, Stern HM, Yue P, Haverty PM, Bourgon R, Zheng J, et al: Diverse somatic mutation patterns and pathway alterations in human cancers. Nature 2010, 466:869-873.

10. Yu J, Mani RS, Cao Q, Brenner CJ, Cao X, Wang X, Wu L, Li J, Hu M, Gong Y, et al: An integrated network of androgen receptor, polycomb, and TMPRSS2-ERG gene fusions in prostate cancer progression. Cancer Cell 2010, 17:443-454.

11. Timmermann B, Kerick M, Roehr C, Fischer A, Isau M, Boerno ST, Wunderlich A, Barmeyer C, Seemann P, Koenig J, et al: Somatic mutation profiles of MSI and MSS colorectal cancer identified by whole exome next generation sequencing and bioinformatics analysis. PLoS One 2010, 5:e15661.

12. Ding L, Ellis MJ, Li S, Larson DE, Chen K, Wallis JW, Harris CC, McLellan MD, Fulton RS, Fulton LL, et al: Genome remodelling in a basal-like breast cancer metastasis and xenograft. Nature 2010, 464:999-1005.

13. Lee W, Jiang Z, Liu J, Haverty PM, Guan Y, Stinson J, Yue P, Zhang Y, Pant KP, Bhatt D, et al: The mutation spectrum revealed by paired genome sequences from a lung cancer patient. Nature 2010, 465:473-477.

14. Ley TJ, Mardis ER, Ding L, Fulton B, McLellan MD, Chen K, Dooling D, Dunford-Shore BH, McGrath S, Hickenbotham M, et al: DNA sequencing of a cytogenetically normal acute myeloid leukaemia genome. Nature 2008, 456:66-72.

15. Shah SP, Morin RD, Khattra J, Prentice L, Pugh T, Burleigh A, Delaney A, Gelmon K, Guliany R, Senz J, et al: Mutational evolution in a lobular breast tumour profiled at single nucleotide resolution. Nature 2009, 461:809-813.

16. Schweiger MR, Kerick M, Timmermann B, Isau M: The power of NGS technologies to delineate the genome organization in cancer: from mutations to structural variations and epigenetic alterations. Cancer Metastasis Rev 2011.

17. Pleasance ED, Cheetham RK, Stephens PJ, McBride DJ, Humphray SJ, Greenman CD, Varela I, Lin ML, Ordonez GR, Bignell GR, et al: A comprehensive catalogue of somatic mutations from a human cancer genome. Nature 2010, 463:191-196.

18. Pleasance ED, Stephens PJ, O'Meara S, McBride DJ, Meynert A, Jones D, Lin ML, Beare D, Lau KW, Greenman C, et al: A small-cell lung cancer genome with complex signatures of tobacco exposure. Nature 2010, 463:184-190.

19. Krawitz PM, Schweiger MR, Rodelsperger C, Marcelis C, Kolsch U, Meisel C, Stephani F, Kinoshita T, Murakami Y, Bauer S, et al: Identity-by-descent filtering of exome sequence data identifies PIGV mutations in hyperphosphatasia mental retardation syndrome. Nat Genet 2010, 42:827-829.

20. Ng SB, Turner EH, Robertson PD, Flygare SD, Bigham AW, Lee C, Shaffer T, Wong M, Bhattacharjee A, Eichler EE, et al: Targeted capture and massively parallel sequencing of 12 human exomes. Nature 2009, 461:272-276. 
21. Okou DT, Steinberg KM, Middle C, Cutler DJ, Albert TJ, Zwick ME: Microarray-based genomic selection for high-throughput resequencing. Nat Methods 2007, 4:907-909.

22. Albert TJ, Molla MN, Muzny DM, Nazareth L, Wheeler D, Song $X$ Richmond TA, Middle CM, Rodesch MJ, Packard CJ, et al: Direct selection of human genomic loci by microarray hybridization. Nat Methods 2007, 4:903-905.

23. Hodges E, Xuan Z, Balija V, Kramer M, Molla MN, Smith SW, Middle CM, Rodesch MJ, Albert TJ, Hannon GJ, McCombie WR: Genome-wide in situ exon capture for selective resequencing. Nat Genet 2007, 39:1522-1527.

24. Porreca GJ, Zhang K, Li JB, Xie B, Austin D, Vassallo SL, LeProust EM, Peck BJ, Emig CJ, Dahl F, et al: Multiplex amplification of large sets of human exons. Nat Methods 2007, 4:931-936

25. Gnirke A, Melnikov A, Maguire J, Rogov P, LeProust EM, Brockman W, Fennell T, Giannoukos G, Fisher S, Russ C, et al: Solution hybrid selection with ultra-long oligonucleotides for massively parallel targeted sequencing. Nat Biotechnol 2009, 27:182-189.

26. Weise A, Timmermann B, Grabherr M, Werber M, Heyn P, Kosyakova N, Liehr T, Neitzel H, Konrat K, Bommer $C$, et al: High-throughput sequencing of microdissected chromosomal regions. Eur J Hum Genet 2010, 18:457-462.

27. Briggs AW, Good JM, Green RE, Krause J, Maricic T, Stenzel U, Lalueza-Fox C, Rudan P, Brajkovic D, Kucan Z, et al: Targeted retrieval and analysis of five Neandertal mtDNA genomes. Science 2009, 325:318-321.

28. Schweiger MR, Kerick M, Timmermann B, Albrecht MW, Borodina T, Parkhomchuk D, Zatloukal K, Lehrach H: Genome-wide massively parallel sequencing of formaldehyde fixed-paraffin embedded (FFPE) tumor tissues for copy-number- and mutation-analysis. PLoS One 2009, 4:e5548,

29. Bartsch G, Horninger W, Klocker H, Pelzer A, Bektic J, Oberaigner W, Schennach H, Schafer G, Frauscher F, Boniol M, et al: Tyrol Prostate Cancer Demonstration Project: early detection, treatment, outcome, incidence and mortality. BJU Int 2008, 101:809-816.

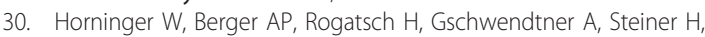
Niescher M, Klocker $H$, Bartsch $\mathrm{G}$ : Characteristics of prostate cancers detected at low PSA levels. Prostate 2004, 58:232-237.

31. Wood HM, Belvedere O, Conway C, Daly C, Chalkley R, Bickerdike M, McKinley C, Egan P, Ross L, Hayward B, et al: Using next-generation sequencing for high resolution multiplex analysis of copy number variation from nanogram quantities of DNA from formalin-fixed paraffinembedded specimens. Nucleic Acids Res 2010, 38:e151.

32. Aird D, Ross MG, Chen WS, Danielsson M, Fennell T, Russ C, Jaffe DB, Nusbaum C, Gnirke A: Analyzing and minimizing PCR amplification bias in Illumina sequencing libraries. Genome Biol 2011, 12:R18.

33. Aihara M, Wheeler TM, Ohori M, Scardino PT: Heterogeneity of prostate cancer in radical prostatectomy specimens. Urology 1994, 43:60-66, discussion 66-67

34. Yatani R, Kusano I, Shiraishi T, Hayashi T, Stemmermann GN: Latent prostatic carcinoma: pathological and epidemiological aspects. Jpn J Clin Oncol 1989, 19:319-326.

35. Sakr WA, Grignon DJ, Crissman JD, Heilbrun LK, Cassin BJ, Pontes JJ, Haas GP: High grade prostatic intraepithelial neoplasia (HGPIN) and prostatic adenocarcinoma between the ages of 20-69: an autopsy study of 249 cases. In Vivo 1994, 8:439-443.

36. Shiraishi T, Watanabe M, Matsuura H, Kusano I, Yatani R, Stemmermann GN: The frequency of latent prostatic carcinoma in young males: the Japanese experience. In Vivo 1994, 8:445-447.

37. Bostwick DG, Shan A, Qian J, Darson M, Maihle NJ, Jenkins RB, Cheng L: Independent origin of multiple foci of prostatic intraepithelial neoplasia: comparison with matched foci of prostate carcinoma. Cancer 1998, 83:1995-2002

38. Macintosh CA, Stower M, Reid N, Maitland NJ: Precise microdissection of human prostate cancers reveals genotypic heterogeneity. Cancer Res 1998, 58:23-28.

39. Mehra R, Han B, Tomlins SA, Wang L, Menon A, Wasco MJ, Shen R, Montie JE, Chinnaiyan AM, Shah RB: Heterogeneity of TMPRSS2 gene rearrangements in multifocal prostate adenocarcinoma: molecular evidence for an independent group of diseases. Cancer Res 2007, 67:7991-7995.

40. Clark J, Attard G, Jhavar S, Flohr P, Reid A, De-Bono J, Eeles R, Scardino P, Cuzick J, Fisher $G$, et al: Complex patterns of ETS gene alteration arise during cancer development in the human prostate. Oncogene 2008, 27:1993-2003.

41. Shah RB, Mehra R, Chinnaiyan AM, Shen R, Ghosh D, Zhou M, Macvicar GR, Varambally S, Harwood J, Bismar TA, et al: Androgen-independent prostate cancer is a heterogeneous group of diseases: lessons from a rapid autopsy program. Cancer Res 2004, 64:9209-9216.

42. Mehra R, Tomlins SA, Yu J, Cao X, Wang L, Menon A, Rubin MA, Pienta K, Shah RB, Chinnaiyan AM: Characterization of TMPRSS2-ETS gene aberrations in androgen-independent metastatic prostate cancer. Cancer Res 2008, 68:3584-3590.

43. Liu W, Laitinen S, Khan S, Vihinen M, Kowalski J, Yu G, Chen L, Ewing CM, Eisenberger MA, Carducci MA, et al: Copy number analysis indicates monoclonal origin of lethal metastatic prostate cancer. Nat Med 2009, 15:559-565.

44. Barry M, Perner S, Demichelis F, Rubin MA: TMPRSS2-ERG fusion heterogeneity in multifocal prostate cancer: clinical and biologic implications. Urology 2007, 70:630-633.

45. Berger MF, Lawrence MS, Demichelis F, Drier Y, Cibulskis K, Sivachenko AY, Sboner A, Esgueva R, Pflueger D, Sougnez C, et al: The genomic complexity of primary human prostate cancer. Nature 470:214-220.

46. Navin N, Krasnitz A, Rodgers L, Cook K, Meth J, Kendall J, Riggs M Eberling $Y$, Troge J, Grubor $V$, et al: Inferring tumor progression from genomic heterogeneity. Genome Res 2010, 20:68-80.

47. Michael FBerger MSL, Francesca Demichelis, Drier KC, Andrey YSivachenko, Andrea Sboner, Esqueva DP, Carrie Sougnez, Robert Onofrio, Scott LCarter Park LH, Lauren Ambrogio, Timothy Fennell, Melissa Parkin, Saksena DV, Alex HRamos, Trevor JPugh, Jane Wilkinson, Fisher WW, Scott Mahan, Kristin Ardlie, Jennifer Baldwin, W. Simons NK, Theresa YMacDonald, Philip WKantoff, Chin SBG, Mark BGerstein, Todd RGolub, Meyerson AT, Eric SLander, Gad Getz, Mark A, Rubin LAG: The genomic complexity of primary human prostate cancer. Nature 2011.

Pre-publication history

The pre-publication history for this paper can be accessed here: http://www.biomedcentral.com/1755-8794/4/68/prepub

doi:10.1186/1755-8794-4-68

Cite this article as: Kerick et al:: Targeted high throughput sequencing in clinical cancer Settings: formaldehyde fixed-paraffin embedded (FFPE) tumor tissues, input amount and tumor heterogeneity. BMC Medical Genomics 2011 4:68.

\section{Submit your next manuscript to BioMed Central and take full advantage of:}

- Convenient online submission

- Thorough peer review

- No space constraints or color figure charges

- Immediate publication on acceptance

- Inclusion in PubMed, CAS, Scopus and Google Scholar

- Research which is freely available for redistribution

Submit your manuscript at www.biomedcentral.com/submit
C Biomed Central 